\title{
IGHV mutational status of nodal marginal zone lymphoma by NGS reveals distinct pathogenic pathways with different prognostic implications
}

\author{
Massimo Granai ${ }^{1} \cdot$ Teresa Amato $^{1} \cdot$ Arianna Di Napoli $^{2} \cdot$ Raffaella Santi $^{3} \cdot$ Federica Vergoni $^{3} \cdot$ Gioia Di Stefano $^{3}$. \\ Virginia Mancini ${ }^{1}$ - Sofya Kovalchuk ${ }^{4}$. Emanuele Cencini ${ }^{5}$ - Alberto Giulio Carta ${ }^{1}$ - Sara Aversa ${ }^{1}$ - Marita Ziepert ${ }^{6}$. \\ Gabriele Cevenini ${ }^{1}$-Stefano Lazzi $^{1}$ - Lorenzo Leoncini $^{1}$ • Cristiana Bellan ${ }^{1}$
}

Received: 22 July 2019 / Revised: 18 October 2019 / Accepted: 29 October 2019/Published online: 4 December 2019

(C) The Author(s) 2020, corrected publication 2020

\begin{abstract}
The precise B cell of origin and molecular pathogenesis of nodal marginal zone lymphoma (NMZL) remain poorly defined. To date, due to the rarity of NMZL, the vast majority of already-published studies have been conducted on a limited number of samples and the technical approach to analyze the immunoglobulin genes was of amplifying rearranged variable region genes with the classical direct sequencing of the PCR products followed by cloning. Here, we studied the B cell Ig heavy-chain repertoires by next-generation sequencing (NGS) in 30 NMZL cases. Most of the cases were mutated $(20 / 28 ; 71.5 \%)$ with homologies to the respective germ line genes ranging from 85 to $97,83 \%$, whereas $8 / 28(28.5 \%)$ were unmutated. In addition, our results show that NMZL cases have a biased usage of specific immunoglobulin heavy-chain variable (IGHV) region genes. Moreover, we documented intraclonal diversity in all (100\%) of the mutated cases and ongoing somatic hypermutations (SHM) have been confirmed by hundreds of reads. We analyzed the mutational pattern to detect and quantify antigen selection pressure and we found a positive selection in 4 cases, whereas in the remaining cases there was an unspecific stimulation. Finally, the disease-specific survival and the progression-free survival were significantly different between cases with mutated and unmutated IGHV genes, pointing out mutational status as a possible new biomarker in NMZL.
\end{abstract}

Keywords BCR $\cdot$ Nodal marginal zone lymphomas $\cdot$ NGS $\cdot$ Clonality analysis

Granai Massimo and Amato Teresa contributed equally to this work.

This article is part of the Topical Collection on Quality in Pathology

Electronic supplementary material The online version of this article (https://doi.org/10.1007/s00428-019-02712-8) contains supplementary material, which is available to authorized users.

Lorenzo Leoncini

lorenzo.leoncini@dbm.unisi.it

1 Department of Medical Biotechnologies, Anatomic Pathology Division, University of Siena, Via delle Scotte, 6, 53100 Siena, Italy

2 Department of Clinical and Molecular Medicine, Pathology Unit, University of Rome "La Sapienza", Rome, Italy

3 Florence Pathology Unit, Careggi University Hospital, Florence, Italy

4 Florence Hematology Unit, University of Florence, Florence, Italy

5 Department of Hematology, University of Siena, Siena, Italy

6 Institute for Medical Informatics, Statistics and Epidemiology, University of Leipzig, Leipzig, Germany

\section{Introduction}

Nodal marginal zone lymphomas (NMZL) represent one of three recognized entities within the category of marginal zone lymphomas (MZL), along with splenic marginal zone lymphomas (SMZL) and extranodal marginal zone lymphomas (ENMZL), with the latter tumors also known as mucosaassociated lymphoid tissue (MALT) lymphomas. NMZL, SMZL, and MALT all belong to the category of indolent small B cell lymphomas [1]. Although NMZL shares many histologic and immunologic features with extranodal MZL of MALT type, clinical characteristics, natural history, and prognosis suggest that nodal MZL should be considered a distinct entity [2].

However, lack of typical markers and absence of a clear consensus for its molecular pathogenesis make the diagnosis of nodal marginal zone lymphoma (NMZL) a problematic subject [3]. Yet, the precise B cell of origin of NMZL remains poorly defined [4]. 
A B cell undergoes germinal center (GC) reaction in response to antigen stimulation, resulting in the generation of a memory B cell with a high specificity and affinity. At the gene level, memory B cells are characterized by somatic mutation (SM) in their rearranged immunoglobulin (Ig) heavy-chain variable $(\mathrm{VH})$ genes [5]. Somatic mutation studies of SMZL and ENMZ have shown that in the great majority of the cases, the tumor cells are of a post-GC, memory B cell derivation, displaying a mutational pattern indicative of positive antigen selection [6].

Because of the rarity of NMZL, it is hard to obtain large study groups, and in all previous studies, the technical approach of amplifying rearranged variable region genes was the classical sequencing methods, i.e., direct sequencing of the PCR products followed by cloning. However, this approach is based on the analysis of limited number of clones that could not be representative for the real intraclonal heterogeneity.

The quantitative nature of next-generation sequencing (NGS) data allows for higher resolution of the subclonal architecture and can be used to decipher mutational signatures and, thus offering a dynamic mechanism for the mutations found in the sample [7].

Here, we studied the B cell Ig heavy-chain repertoires to characterize the diversity of the heavy-chain CDR3 region and the constituent $\mathrm{V}, \mathrm{D}$, and $\mathrm{J}$ segments that comprise it, in 30 NMZL cases to acquire insight into the nature of its cell of origin and to identify mutation patterns reminiscent of antigen selection processes. Our results show that NMZL cells have a biased usage of IGHV genes in favor of specific segments. We also shed light on the role of antigenic stimulation in the aetiology of NMZL and in the maintenance of BCR integrity. In addition, the postulated normal counterpart of this lymphoma consists of specific B lymphocyte subsets, with cases carrying unmutated and mutated IGHV genes which impact the clinical outcome as observed in chronic B cell leukemia (BCLL) and other small B cell lymphomas.

\section{Materials and methods}

\section{Patients and tissues samples}

Thirty NMZL formalin-fixed paraffin-embedded (FFPE) cases were selected from the files of the Department of Medical Biotechnologies, University of Siena; Pathology Unit, Careggi University Hospital, Florence; and Department of Pathology, La Sapienza University, Rome. In all the cases, the diagnosis of NMZL was performed primarily on lymph node localization in the absence of previous or concurrent involvement of any extranodal site, with the exception of bone marrow. All the cases were reviewed by expert hematopathologists by morphological and immunohistochemical criteria according to WHO classification. In addition, to rule out a possible misdiagnosis of lymphoplasmacytic lymphoma, all cases were analyzed for MYD88 L265P mutation and two cases carrying the mutation of this gene were excluded from the study $[8,9]$. As further validation of NMZL diagnosis, we also demonstrated the absence of glycosylation motifs in the VDJ regions of all the analyzed cases, hence excluding concealed follicular lymphomas [10].

\section{PCR amplification and high-throughput sequencing by Roche 454 GS Junior instrument}

Genomic DNA was extracted from 5 to $10 \mu \mathrm{m}$ of FFPE tissue using a DNA extractor (MagCore NucleicAcid Extractor, RBC Bioscience, Taiwan) and MagCore Genomic DNA FFPE One-Step Kit, following the manufacturer's recommendations. Genomic DNA quality was assessed using BIOMED2 control gene PCR protocol and samples with a DNA product size of $\geq 300$ base pairs (bp) were analyzed [11]. Before initiating VDJ gene rearrangement analysis by HTS, all cases were analyzed to evaluate clonality according to the BIOMED-2 protocol [11]. NGS analysis was performed on 454 GS Junior system (Roche) previously described [12]. Data analysis was performed using the Roche (Basel, Switzerland) proprietary software package for the 454 GS Junior system (Roche). Image acquisition, image processing, and signal processing were performed during the run.

\section{Bioinformatical analysis}

The bioinformatical analysis was performed by using the 454 GS Junior system, as previously described [12].

\section{Sequence data analysis}

To determine the IGHV, IGHD, and IGHJ gene usage and the mutational status of each IGHV gene, sequences were submitted to the international ImmunoGeneTics (IMGT, Montpellier, France) database $[13,14]$ and aligned to the closest matching germ line gene by using the IMGT/V-QUEST and IMGT/ Junction Analysis software [15, 16], as previously described [12].

\section{Clustering of VH CDR3 sequences}

The length of the VH CDR3 of the immunoglobulin heavychain gene rearrangement was computed using the IMGT database starting from the first codon after the conserved cysteine up to the position preceding the conserved tryptophan of the $\mathrm{JH}$ gene segment, as previously described [17-19]. 


\section{Antigen selection}

We used a recently published tool known as BASELINE (i.e., Bayesian estimation of antigen-driven selection; http://clip.med. yale.edu/selection) to detect and quantify antigen selection in individual or multiple sequences based on mutational patterns, normalized to germ line sequences, and provided a visual representation of differences in selective pressure [20, 21]. Clonally related sequences and productive heavy-chain V-region sequences (CDR1-FWR2-CDR2-FWR3) were analyzed using BASELINE version $1.3(01 / 30 / 2014)$.

Typical antigen-driven activation results in positive selection in the complementary-determined regions (CDRs), which directly interact with antigen, and negative selection in the framework regions (FRs), which are more important for structural integrity. Patterns of selective pressure contrary to this model indicate non-specific activation [22].

\section{Statistical analysis}

A multivariate analysis based on Cox's proportional hazards regression was performed to verify the potential relationship between survival of the patients, mutational status, and critical clinical parameters (e.g., age, ECOG, LDH, stage, and therapeutic regimen) [23].

Survival curves were plotted using the Kaplan-Meier method and were compared using log-rank test. According to Cheson et al., overall survival (OS) was defined as the time from diagnosis to death; patients who remained alive were censored at the last date of follow-up [24]. Progression-free survival (PFS) was defined as the time from diagnosis to the date of first documented recurrence. Disease-specific (or disease-related) survival (DSS) was calculated from the date of diagnosis until the patient's death due to the NMZL. Statistical analysis was performed using SPSS software version 20.0 [25]. For all the tests, $p<0.05$ (two-sided) was considered statistically significant.

\section{Results}

\section{Histopathological and immunophenotypic features}

Most of the cases were characterized by a parafollicular and/or interfollicular infiltrate of neoplastic cells effacing the lymph node architecture and, to a considerably lesser extent, regressed residual lymphoid follicles, lacking well-formed germinal centers with attenuated mantle cuffs. The neoplastic cells were heterogeneous in appearance with monocytoid, centrocyte-like blastic and plasmacytoid features. All of the cases expressed pan-B cell markers (CD20, PAX5). Moreover, CD23 was also negative in the vast majority of the cases $(21 / 28 ; 75 \%)$. CD21 showed a disrupted and expanded residual meshwork. All of the cases were negative for CD5 and cyclin D1. Germinal center markers (CD10, BCL6) were likewise negative. IgD IHC was also negative where performed. Conversely, IgM IHC, when available, was positive.

\section{High-throughput sequencing analysis of IGHV gene repertoire in NMZL}

A total of 180,050 reads were generated. During the platformspecific processing, 70,904 reads failed the filtering process owing to missing or incomplete barcodes. For our 28 samples, 109,146 reads were obtained as final 454 output with an average depth of 2831 reads, with a minimum and maximum depth of 808 and 16,114 reads respectively. Unproductive rearrangements were excluded from analysis.

The IGHV, IGHD, and IGHJ gene and allele usage were obtained using the statistical analysis of IMGT/HighVQUEST available online. This analysis is performed automatically on the "1 copy"| "single allele" (for V, D, and J) category. All the 28 cases were clonal on NGS using the criterion that a clonal cluster(s) must beat least fourfold more abundant than the largest clonotype of the background $[12,26]$. In particular, the presumed monoclonal clusters, represented from 20 to $99 \%$ of the total reads, confirm the results of GeneScan profiles ranging from clonal to clonal with polyclonal background according to BIOMED-2 criteria. When all the sequences were aligned with IMGT tools for nucleotide analysis of immunoglobulin (IG), polymorphisms, and IG mutations, clusters showing identical IGHV, IGHD, and IGHJ usage and CDR3 regions as the presumed monoclonal clusters were detected. All the results representative of clonotypes AA (amino acid) identified by NGS were overlapped and confirmed with the results obtained by Sanger sequencing.

Most of the cases were mutated $(20 / 28 ; 71.5 \%)$ (MNMZL) with homologies to the respective germ line genes ranging from 85 to $97,83 \%$, whereas $8 / 28$ (28.5\%) were unmutated (U-NMZL) (Fig. 1).

We demonstrated intraclonal diversity (ID) in all (100\%) the patients with a mutated IGHV; ongoing SHM have been confirmed by hundreds of reads. Detailed results are reported in Supplementary Table 1. Subclones have been identified with a mean of 2-6 subclones per case. Figure 2 illustrates an example of the branching of the lymphoma clone and shows that distinct subclones evolved along similar, although separate pathways.

Nineteen productive heavy-chain V-region clonally related sequences were evaluated for selection pressure (Supplementary Table 1). The range of mutations was 30-7 with a V-region germ line identity\% range 85.00-96.85. The BASELINE method found positive selection in the CDRs and negative selection in the FRWS of the heavy chains in 4 patients indicating selective pressure by antigens. In the 
Fig. 1 Gene usage in mutated vs unmutated NMZL. 6 out of 28 cases (21.4\%) utilized VH1-69 gene (3 mutated; 3 unmutated), 5 out of $28(17.8 \%)$ were most homologous to a $\mathrm{VH} 1-2$ gene segment ( 3 mutated; 2

unmutated), and 4 of $28(14.2 \%)$ were most homologous to a $\mathrm{VH} 3$ 7 gene segment (3 mutated; 1 unmutated) while the remaining 13 were most closely related to different $\mathrm{VH}$ genes from the $\mathrm{VH} 2$ family (VH2-5), VH3 family( VH3-23, VH3-30, VH3-33, VH348), VH4 family (VH4-31, VH434, VH4-59), and VH6 family (VH6-1)

\section{IGHV Usage and frequency in Mutated Vs. Unmutated NMZL}

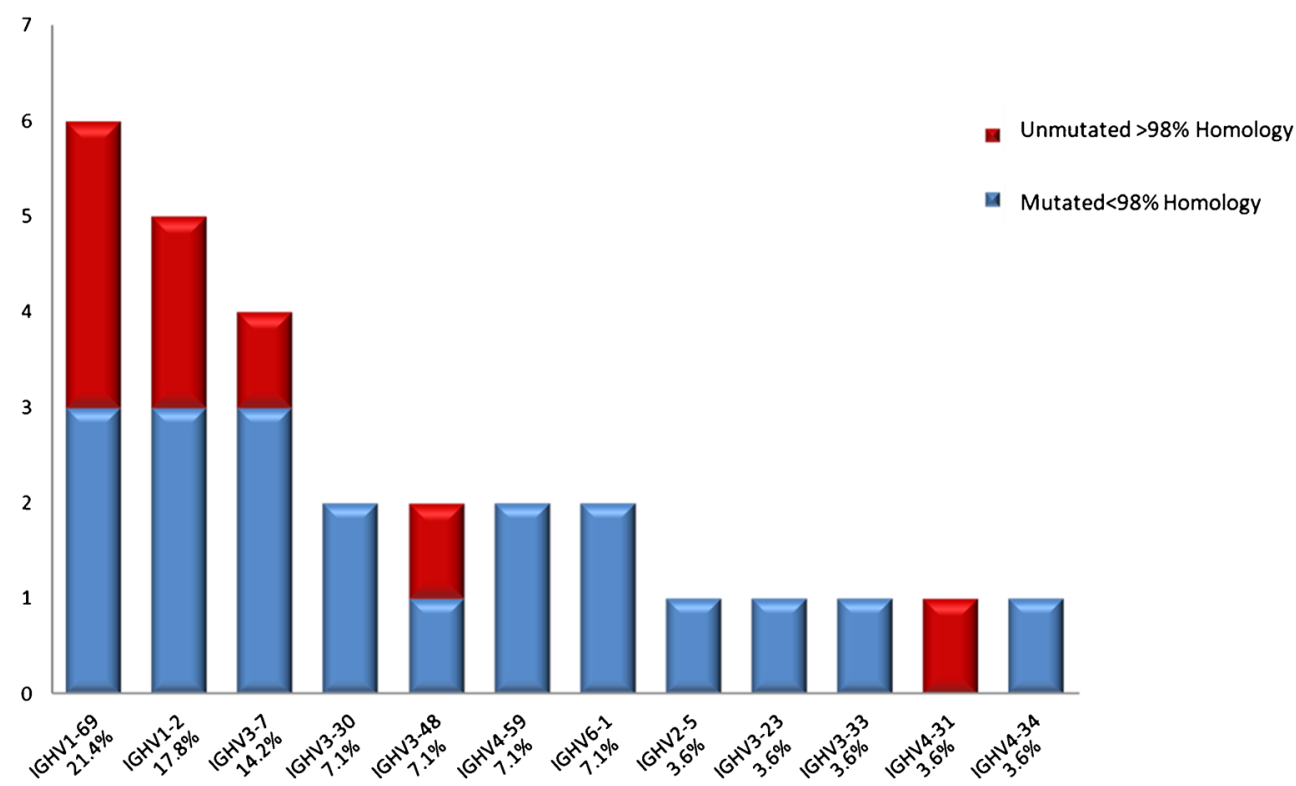

remaining cases, we observed only a negative selection in the FRWS indicating a non-specific activation by the antigen/ antigens to maintain the structural conservation and integrity of BCR [27] (Supplementary Table 2; Supplementary Figure 1).

\section{Analysis of IGHV Gene Usage}

Clonal and in-frame VH gene sequences from NMZL cases derived from VH1, VH2, VH3, VH4, and VH6 families and were further stratified according to the mutational status. In

\section{NMZL : IGHVDJ rearrangement with ID and branching process}

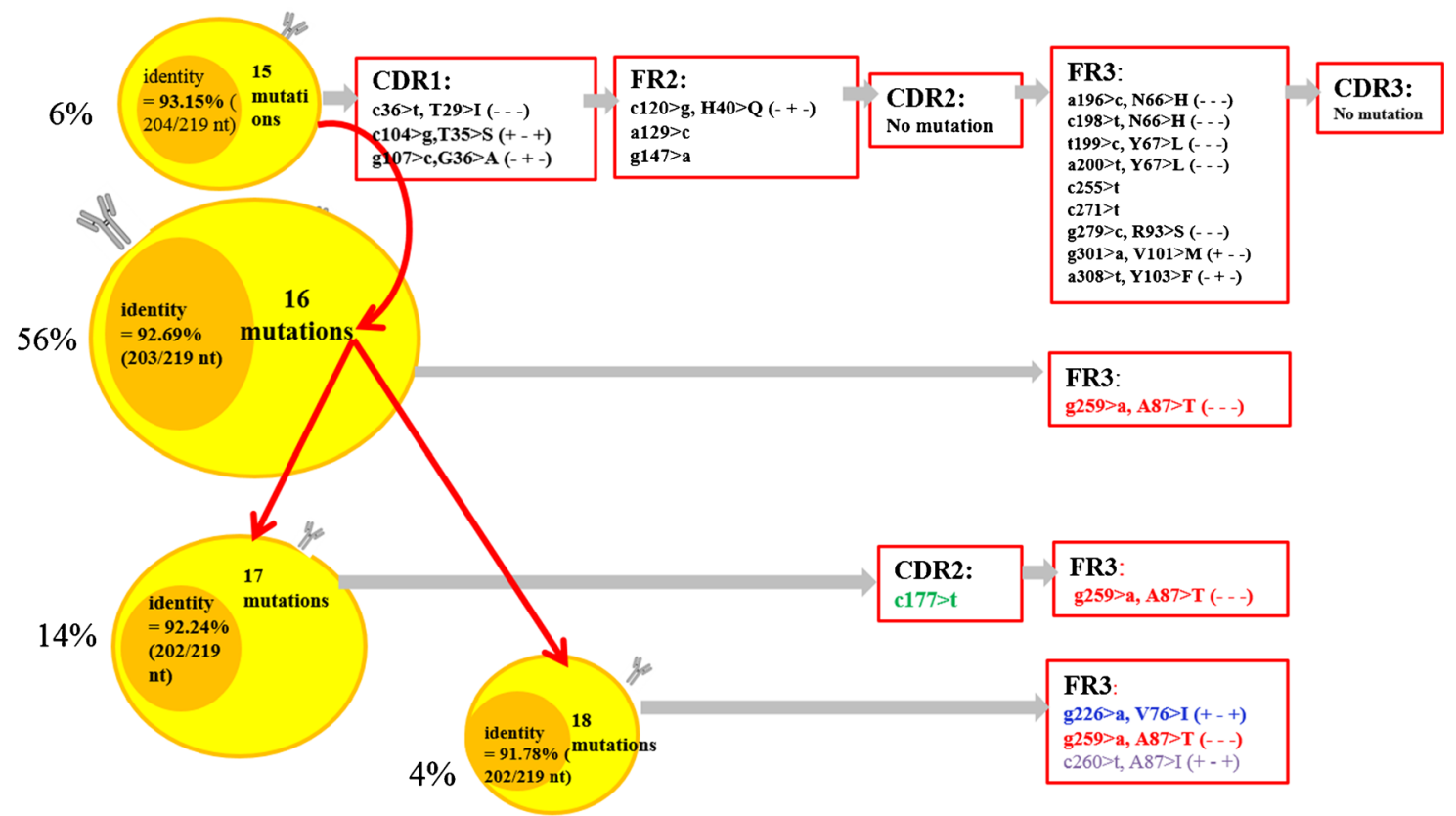

Fig. 2 Example of branching of a lymphoma clone. The four different clusters represented show identical IGHV, IGHD, and IGHJ usage and related CDR3 regions but exhibit different somatic mutations. The mutations indicated in black color in the box are common mutations, while those in red, blue, and green are ongoing mutations. The dominant clone is confirmed by $56 \%$ of the sequences of clonotype AA and carried 16 mutations. The other three minor clones are represented by $6 \%, 14 \%$, and $4 \%$ of the sequences of the clonotype AA. Respectively, all of the clones shared common mutations of the first clone $(6 \%$ of sequences) 
particular, 6 out of 28 cases (21.4\%) utilized the same VH1-69 gene (3 mutated; 3 unmutated), 5 out of 28 (17.8\%) were most homologous to a VH1-2 gene segment (3 mutated; 2 unmutated), and 4 of 28 (14.2\%) were most homologous to a VH3-7 gene segment (3 mutated; 1 unmutated) while the remaining 13 were most closely related to different $\mathrm{VH}$ genes from the $\mathrm{VH} 2$ family ( $V H 2-5)$, VH3 family ( $V H 3-23, V H 3-30$, VH3-33, VH3-48), VH4 family (VH4-31, VH4-34, VH4-59), and VH6 family (VH6-1) (Fig. 1).

In addition, we also determined the IGHD and IGHJ genes used in IGHV-D-J sequences analyzed. The IGHD genes used were IGHD3 (35.7\%), IGHD1 (21.4\%), and IGHD2 (14.2\%) and several others to a much lower extent, including IGHD4, IGHD5, and IGHD6. The IGHJ gene usage in all cases showed that IGHJ4 (46.4\%) was used the most, followed by IGHJ6 (35.7\%), IGHJ3 (3.57\%), and IGHJ5 (7.1\%).

The average VH CDR3 length of NMZL cells was 15, 7 AA ranging from 8 to 23 residues. In addition, we compared the CDR3 regions of the NMZL cases to previously published cases of CLL and SMZL that used the same VH region, and they differ in length and AA composition $[6,17,18]$.

\section{Pattern of progression and survival}

After a median follow-up of 5 years, no patient had developed splenic or MALT involvement during the course of disease. Additional clinical information is summarized in Table 1.

At the time of the analysis, 12 patients were deceased. Death related to lymphoma occurred in 5/28 patients. Relapse of disease occurred in 10 patients. Global median

Table 1 Summarized clinical features with therapy of 28 NMZL cases

\begin{tabular}{lll}
\hline & $N /$ tot & Percent \\
\hline Clinical features & & \\
Male & $15 / 28$ & 53 \\
Median age (years) & 67 & \\
Age $>60$ (years) & $22 / 28$ & 78 \\
Peripheral lymph nodes & $20 / 28$ & 71 \\
Abdominal/thoracic lymph nodes & $8 / 28$ & 29 \\
Bone marrow involvement & $11 / 28$ & 40 \\
Liver involvement & $0 / 28$ & 0 \\
Spleen involvement & $0 / 28$ & 0 \\
Pleural localization & $0 / 28$ & 0 \\
LDH normal & $23 / 28$ & 82 \\
ECOG $\geq 2$ & $1 / 28$ & 3 \\
Global median OS (months) & 66 & $(95 \%$ CI 52.9-79.0) \\
Treatments & & \\
Watch and wait & $6 / 28$ & 21 \\
R-Bendamustine & $18 / 28$ & 3 \\
R-Leukeran, R-FC & $4 / 28$ & 14 \\
\hline
\end{tabular}

$R-F C$ rituximab-fludarabine-cyclophosphamide time of overall survival (OS) was 66 months (95\% CI 52.9 79.0).

Despite the low sample size, we applied the multivariate Cox survival analysis. At univariate analysis, ECOG ( $p$ $0.035)$, IGHV status $(p$ 0.005) with LDH $(p 0.026)$, and IGHV status ( $p$ 0.0002) were respectively significant for OS, DSS, and PFS (Supplementary Table 3). However, elevated LDH and ECOG were infrequent (18\% and 3\%, respectively). Considering all the bivariate combinations, only the mutational status remains significant in DSS and PFS analyses. Cox models with higher dimensionality were completely not statistically significant. Therefore, the mutational status showed to be an independent factor affecting survival and consequently clinical variables did not significantly affect survival or acted as adjustment factors changing the mutational status contribution. Accordingly, patients were stratified on the grounds of IGHV mutational status. A median time of overall survival of 62 months (95\% CI 46.5-77.5) and 72 months (95\% CI 49.6-94.4) was shown for U-NMZL and M-NMZL patients, respectively. However, Kaplan-Meier survival curves for OS (Fig. 3a) showed a non-statistically significant difference between unmutated and mutated patients $(p=0.18)$. Interestingly, disease-specific survival (Fig. 3b) and progression-free survival (Fig. 3c) both exhibited a high significant difference between the two groups $(p<0.01)$. In particular, for the unmutated patients, the median times of DSS and PFS were 66 months (95\% CI 48.9-83.1) and 36 months (95\% CI 16.3-55.7), respectively.

\section{Discussion}

Analyses of antigen-receptor genes in human lymphoma represent a useful tool in understanding their pathogenesis and clonal history [7].

Somatic hypermutations seem to be restricted to B cells proliferating within the microenvironment of the germinal center (GC). As a consequence, the presence of somatic mutations in the variable region of the rearranged immunoglobulin genes is actually considered the hallmark of B cells that have participated in a GC reaction [28]. Moreover, the pattern of the distribution of somatic mutations and a preferential usage of immunoglobulin variable, diversity, and joining segments may reveal a role of antigens in driving B cell proliferation. Clustering of nucleotide mutations leading to an amino acid substitution in the CDRs of VH segments is considered to indicate that the hypermutation process is driven by an antigen [22].

Here we show that in NMZL cases, the VH1 family genes were significantly overrepresented compared with transitional B cells, naive B cells, and IgM memory B cells [29]. In particular, our data are in accordance with previous studies which showed a biased usage of the $I G H V$ genes in favor of $I G H V-1$ - 

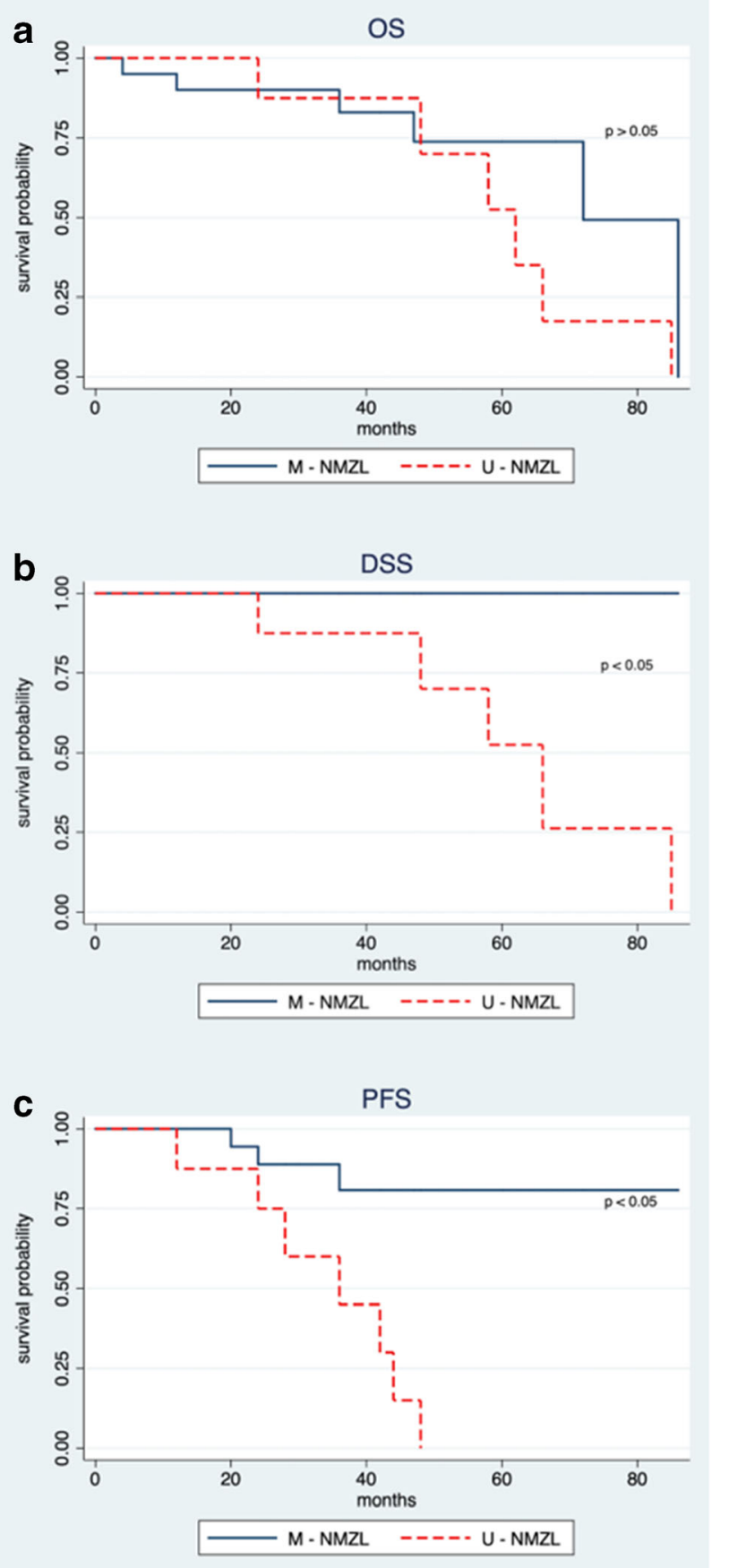

Fig. 3 Survival analysis for NMZL according to the mutational status. Kaplan-Meier survival curves for overall survival in U-NMZL and MNMZL patients $(p=0.18)$ (a). Kaplan-Meier survival curves for diseasespecific survival in U-NMZL and M-NMZL patients $(p<0.01)(\mathbf{b})$. Kaplan-Meier survival curves for progression-free survival in U-NMZL and M-NMZL patients $(p<0.01)(\mathbf{c})$

69 ( 6 out of 28 cases; 21.4\%) [30]. Conversely, we found an overrepresentation of $\mathrm{VHI}-2$ gene in our cohort of cases ( 5 out of $28 ; 17.8 \%$ ) which has been found mainly in SMZL [30]. $\mathrm{VH1}-2$ is known to react with antigen exposed on apoptotic cells, suggesting that at least a subset of NMZL may arise from a self-antigen antibody producing B cell [6]. Furthermore, VH1-69 gene segment is the most used in hepatitis $\mathrm{C}$ virus-positive NMZL [31]. However, the use of VHI69 gene in our series is not restricted to hepatitis $\mathrm{C}$ virus infection. In fact, VH1-69 utilizing antibodies are also found in protective antibody responses to additional viral pathogens such as influenza infection, respiratory syncytial virus infection, and HIV-1 [32]. On the other hand, we could not confirm in this series an overrepresentation of $\mathrm{VH} 4-34$, as reported in previous studies [30, 33].

In line with previous studies, 20 out of 28 cases $(71.5 \%)$ carried SHM in their immunoglobulin genes [30]. We confirmed ID in all the mutated NMZL subgroup of patients analyzed, supporting the notion that the SHM mechanism remains active post-transformation and outside the germinal centers, further diversifying the clonotypic IG receptors. Therefore, the finding of ongoing mutations as indicated by intraclonal variations in NMZL provides the genetic evidence that the tumor responds to antigen stimulation, which may play an important role in its clonal expansion [34]. Several other studies have also demonstrated germinal centerindependent SHM. In particular, Warsame et al. showed evidence of ongoing mutations in micro dissected monocytoid $\mathrm{B}$ cells and expression of activation-induced cytidine deaminase (AID) which is required for SHM [35]. However, according to the BASELINE method, we found a positive selection only in 4 cases, whereas in the remaining cases, there was an unspecific antigenic stimulation that might reflect the necessity of preserving the integrity of BCR enabling the neoplastic cells to avoid apoptotic death [27]. Thus, this finding implies that the presence of BCR itself is necessary to generate a survival signal in the malignant cells.

Taking into account all of the above findings, the obvious conclusion is that environmentally encountered antigen plays at least some part in the maintenance of neoplastic phenotype in NMZL. Hence, immunogenic and functional evidence supports a role for antigen in the natural history of a subset of NMZL. However, the timing and duration of antigen interactions and their relevance for evolution of the disease remain elusive.

In addition, oncogenic events contribute to lymphoma growth and progression and may represent the first step of malignant transformation as demonstrated in recent genomic studies. Consistent with the physiological involvement of NOTCH, NF-KB, B cell receptor, and toll-like receptor signalling in the differentiation of mature $\mathrm{B}$ cells into the marginal zone B cells, many oncogenic mutations of genes involved in these pathways have been identified in MZL [36, 37]. In particular, although the NMZL genetic signature largely overlaps with SMNL, somatic coding-sequence mutations and deletions of the receptor-type tyrosine phosphatase gene PTPRD have been identified as a molecular feature of NMZL among indolent B cell tumors [2]. 
Interestingly, a subset of our cases (28.5\%) did not carry SHM. The existence of unmutated IGHV genes could mean that the transformation leading to NMZL does not target exclusively post-germinal center B cells that bear SHM and have been submitted to T-dependent antigen selection. Conversely, U-NMZL may represent a subgroup not arising from postgerminal center B cells with a different pathogenesis which originates from a cell that has maturated outside of the germinal center and still maintains a naive-like epigenetic signature. Indeed, the possible presence of both virgin B cells and hypermutated B cells in NMZL suggests different modalities for the recruitment of B cells in the marginal zone [38]. Thus, in accordance with previous studies, the observed pattern of $\mathrm{V}_{\mathrm{H}}$ mutations suggests that NMZL may originate from different subsets of marginal zone B cells: the naive B cells that express unmutated $\mathrm{V}_{\mathrm{H}}$ genes and memory $\mathrm{B}$ cells characterized by somatic mutations [34].

The molecular heterogeneity that characterizes NMZL may thus reflect two molecular subtypes of the disease with two different cells of origin. The analysis of IGHV genes of other B cell lymphomas, including chronic lymphocytic leukemia (CLL), splenic marginal zone lymphoma (SMZL), and mantle cell lymphoma (MCL), has also revealed an unexpected heterogeneity in mutational status [39]. This heterogeneity has also been related to prognosis particularly in CLL, in which IGHV sequence analysis has become widely used for the purpose of prognostication [40, 41]. No international prognostic scoring system is available for NMZL and the value of biomarkers in NMZL remains unclear because of the small size of the series, heterogeneity of treatment, and lack of prospective clinical trials [17-45]. According to our knowledge, this is the first report which points out at the mutational status of the immunoglobulin genes as a prognostic biomarker for stratifying NMZL patients. In fact, cases characterized by unmutated immunoglobulin genes show a more aggressive clinical course. In particular, the disease-specific survival and the progression-free survival were significantly different between cases with mutated or unmutated IGHV genes. However, due to a limited number of cases, our results need to be confirmed in additional series of patients, possibly in prospective clinical trials, before applied in clinical practice.

On the other hand, we did not detect a correlation between the usage of a specific $\mathrm{VH}$ gene with survival probability. Further studies with larger populations will be needed to determine whether there is an association between $\mathrm{VH}$ gene usage and prognosis and whether there is a parallel or not with CLL.

In summary, we have shown that NMZL cells show a biased usage of IGHV genes in favor of specific segments and the role of antigenic stimulation in the aetiology of NMZL by maintaining BCR integrity. In addition, the postulated normal counterpart of this lymphoma consists of specific B lymphocyte subsets, with unmutated and mutated IGHV genes, expanding the overlap among small B cell lymphomas in terms of cell of origin and clinical outcome.
Authors' contribution All individuals listed as co-authors of the manuscript (Granai Massimo and Amato Teresa) designed the work and acquired, analyzed, and interpreted the data. Di Napoli A designed the work and acquired the data. All co-authors (Lazzi Stefano, Santi Raffaella, Vergoni Federica, Di Stefano Gioia, Mancini Virginia, Aversa Sara, Kovalchuk Sofya, Cencini Emanuele, Marita Ziepert, Cevenini Gabriele, and Carta Giulio Alberto) acquired and analyzed the data. Leoncini Lorenzo and Bellan Cristiana gave the final approval of the version to be published and agreed to be accountable for all aspects of the work in ensuring that questions related to the accuracy or integrity of any part of the work be appropriately investigated and resolved.

\section{Compliance with ethical standards}

Conflict of interest The authors declare that they have no conflict of interest.

Ethical approval All procedures performed in studies involving human participants were in accordance with the ethical standards of the institutional research committee and with the 1964 Helsinki declaration and its later amendments or comparable ethical standards. This was a noninterventional study on archived tissue samples.

Open Access This article is licensed under a Creative Commons Attribution 4.0 International License, which permits use, sharing, adaptation, distribution and reproduction in any medium or format, as long as you give appropriate credit to the original author(s) and the source, provide a link to the Creative Commons licence, and indicate if changes were made. The images or other third party material in this article are included in the article's Creative Commons licence, unless indicated otherwise in a credit line to the material. If material is not included in the article's Creative Commons licence and your intended use is not permitted by statutory regulation or exceeds the permitted use, you will need to obtain permission directly from the copyright holder. To view a copy of this licence, visit http://creativecommons.org/licenses/by/4.0/.

\section{References}

1. Swerdlow SH, Campo E, Harris NL et al (2017) WHO classification of tumours of haematopoietic and lymphoid tissues (revised 4th Ed, Vol 2). IARC, Lyon

2. Bertoni F, Rossi D, Zucca E (2018) Recent advances in understanding the biology of marginal zone lymphoma. F1000Res 7:406

3. Raderer M (2017) The multiple faces of marginal zone lymphomas. Hematol Oncol 35(Suppl 1):46-48

4. Pileri S, Ponzoni M (2017) Pathology of nodal marginal zone lymphomas. Best Pract Res Clin Haematol. Best Pract Res Clin Haematol 30(1-2):50-55

5. Seifert M, Küppers R (2016) Human memory B cells. Leukemia 30(12):2283-2292

6. Bikos V, Karypidou M, Stalika E et al (2016) An immunogenetic signature of ongoing antigen interactions in splenic marginal zone lymphoma expressing IGHV1-2*04 receptors. Clin Cancer Res 22(8):2032-2040

7. Ohgami R, Rosenwald A, Bagg A (2018) Next-generation sequencing for lymphomas. J Mol Diagn 20(2):163-165

8. Schmidt J et al (2015) MYD88 L265P and CXCR4 mutations in lymphoplasmacytic lymphoma identify cases with high disease activity. Br J Haematol 169(6):795-803

9. Swerdlow S, Kuzu I, Dogan A et al (2016) The many faces of small B cell lymphomas with plasmacytic differentiation and the contribution of MYD88 testing. Virchows Arch 468(3):259-275 
10. Kosmidis P, Bonzheim I, Dufke C et al (2017) Next generation sequencing of the clonal IGH rearrangement detects ongoing mutations and interfollicular trafficking in in situ follicular neoplasia. PLoS One 12(6): 0178503

11. van Dongen J, Langerak A, Brüggemann M et al (2003) Design and standardization of PCR primers and protocols for detection of clonal immunoglobulin and T-cell receptor gene recombinations in suspect lymphoproliferations: report of the BIOMED-2 Concerted Action BMH4-CT98-3936. Leukemia 17(12):2257-2317

12. Amato T, Abate F, Piccaluga $P$ et al (2016) Clonality analysis of immunoglobulin gene rearrangement by next-generation sequencing in endemic Burkitt lymphoma suggests antigen drive activation of BCR as opposed to sporadic Burkitt lymphoma. Am J Clin Pathol 145:116-127

13. Alamyar E, Duroux P, Lefranc M et al (2012) IMGT(®) tools for the nucleotide analysis of immunoglobulin (IG) and T cell receptor (TR) V-(D)-J repertoires, polymorphisms, and IG mutations: IMGT/V-QUEST and IMGT/highv-QUEST for NGS. Methods Mol Biol 882:569-604

14. Lefranc M, Giudicelli V, Ginestoux C et al (2009) IMGT, the international immunogenetics information system. Nucleic Acids Res 37:D1006-D1012

15. Ambrosio M, Rocca B, Ginori A et al (2015) A look into the evolution of Epstein-Barr virus-induced lymphoproliferative disorders: a case study. Am J Clin Pathol 144:817-822

16. Ambrosio M, De Falco G, Rocca B et al (2015) Langerhans cell sarcoma following marginal zone lymphoma: expanding the knowledge on mature B cell plasticity. Virchows Arch 467:471-480

17. Murray F, Darzentas N, Hadzidimitriou A et al (2008) Stereotyped patterns of somatic hypermutation in subsets of patients with chronic lymphocytic leukemia: implications for the role of antigen selection in leukemogenesis. Blood 111:1524-1533

18. Stamatopoulos K, Belessi C, Moreno C et al (2007) Over $20 \%$ of patients with chronic lymphocytic leukemia carry stereotyped receptors: pathogenetic implications and clinical correlations. Blood 109:259-270

19. Bomben R, Dal Bo M, Capello D et al (2007) Comprehensive characterization of IGHV3-21-expressing B-cell chronic lymphocytic leukemia: an Italian multicenter study. Blood 109:2989-2998

20. Yaari G, Uduman M, Kleinstein S (2012) Quantifying selection in high-throughput Immunoglobulin sequencing data sets. Nucleic Acids Res 40(17):e134

21. Uduman M, Yaari G, Hershberg U et al (2011) Detecting selection in immunoglobulin sequences. Nucleic Acids Res 39:W499-W504

22. Bose B, Sinha S (2005) Problems in using statistical analysis of replacement and silent mutations in antibody genes for determining antigen-driven affinity selection. Immunology 116(2):172-183

23. Thieblemont C, Cascione L, Conconi A et al (2017) A MALT lymphoma prognostic index. Blood 130(12):1409-1417

24. Cheson B, Fisher R, Barrington S et al (2014) Recommendations for initial evaluation, staging, and response assessment of Hodgkin and non-Hodgkin lymphoma: the Lugano classification. J Clin Oncol 32(27):3059-3068

25. IBM Corp. IBM SPSS Statistics for Windows, Version 20.0. Armonk, NY. Released 2011.

26. Schumacher J, Duncavage E, Mosbruger T et al (2014) A comparison of deep sequencing of TCRG rearrangements vs traditional capillary electrophoresis for assessment of clonality in T-Cell lymphoproliferative disorders. Am J Clin Pathol 141(3):348-359

27. Yam-Puc J, Zhang L, Zhang Y, Toellner K (2018) Role of B-cell receptors for B-cell development and antigen-induced differentiation. F1000Res 7:429

28. Methot S, Di Noia J (2017) Molecular mechanisms of somatic hypermutation and class switch recombination. Adv Immunol $133: 37-87$
29. Martin V, Wu Y, Townsend C et al (2016) Transitional B cells in early human B cell development - time to revisit the paradigm? Front Immunol 7:546

30. Xochelli A, Bikos V, Polychronidou E et al (2019) Disease-biased and shared characteristics of the immunoglobulin gene repertoires in marginal zone B cell lymphoproliferations. J Pathol 247:416-421

31. Marasca R, Vaccari P, Luppi M et al (2001) Immunoglobulin gene mutations and frequent use of VH1-69 and VH4-34 segments in hepatitis $\mathrm{C}$ virus-positive and hepatitis $\mathrm{C}$ virus-negative nodal marginal zone B-cell lymphoma. Am J Pathol 159(1):253-261

32. Chen F, Tzarum N, Wilson I et al (2019) VH1-69 antiviral broadly neutralizing antibodies: genetics, structures, and relevance to rational vaccine design. Curr Opin Virol 34:149-159

33. Traverse-Glehen A, Davi F, Ben Simon E et al (2005) Analysis of VH genes in marginal zone lymphoma reveals marked heterogeneity between splenic and nodal tumors and suggests the existence of clonal selection. Haematologica 90(4):470-478

34. Conconi A, Bertoni F, Pedrinis E et al (2001) Nodal marginal zone B-cell lymphomas may arise from different subsets of marginal zone B lymphocytes. Blood 98(3):781-786

35. Warsame A, Delabie J, Malecka A et al (2012) Monocytoid B cells: an enigmatic B cell subset showing evidence of extrafollicular immunoglobulin gene somatic hypermutation. Scand J Immunol 75(5):500-509

36. Spina V, Khiabanian H, Messina $M$ et al (2016) The genetics of nodal marginal zone lymphoma. Blood 128(10):1362-1373

37. Agathangelidis A, Xochelli A, Stamatopoulos K et al (2017) A gene is known by the company it keeps: enrichment of TNFAIP3 gene aberrations in MALT lymphomas expressing IGHV4-34 antigen receptors. J Pathol 243(4):403-406

38. Stein K, Hummel M, Korbjuhn P et al (1999) Monocytoid B cells are distinct from splenic marginal zone cells and commonly derive from unmutated naive $\mathrm{B}$ cells and less frequently from postgerminal center B cells by polyclonal transformation. Blood 94(8):2800 2808

39. Puente X, Jares P, Campo E (2018) Chronic lymphocytic leukemia and mantle cell lymphoma: crossroads of genetic and microenvironment interactions. Blood 131(21):2283-2296

40. Amato T, Sall A, Dièye T et al (2017) Preferential usage of specific immunoglobulin heavy chain variable region genes with unmutated profile and advanced stage at presentation are common features in patients with chronic lymphocytic leukemia from Senegal. Am J Clin Pathol 148(6):545-554

41. Del Giudice I, Foà R (2019) Another step forward in the 20-year history of IGHV mutations in chronic lymphocytic leukemia. Haematologica 104(2):219-221

42. Thieblemont C (2017) Improved biological insight and influence on management in indolent lymphoma. Talk 3: update on nodal and splenic marginal zone lymphoma. Hematology Am Soc Hematol Educ Program 2017(1):371-378

43. Tadmor T, Polliack A (2017) Nodal marginal zone lymphoma: clinical features, diagnosis, management and treatment. Best Pract Res Clin Haematol 30(1-2):92-98

44. Arcaini L, Lucioni M, Boveri E, Paulli M (2009 Sep) Nodal marginal zone lymphoma: current knowledge and future directions of an heterogeneous disease. Eur J Haematol 83(3):165-174

45. van den Brand M, van der Velden WJ, Diets IJ, Ector GI, de Haan AF, Stevens WB, Hebeda KM, Groenen PJ, van Krieken HJ (2016) Clinical features of patients with nodal marginal zone lymphoma compared to follicular lymphoma: similar presentation, but differences in prognostic factors and rate of transformation. Leuk Lymphoma 57(7):1649-1656

Publisher's note Springer Nature remains neutral with regard to jurisdictional claims in published maps and institutional affiliations. 